2. Морозова Е.А. Демографическая ситуация и ее влияние на социально-экономическое развитие региона / Е.А. Морозова, А.Н. Челомбитко, Л.М.Андреева. Вестник Кемеровского государственного университета, - 2012. $219 \mathrm{c}$.

3. Плотникова В.В. Концептуальные подходы к анализу эффективности деятельности холдинговых структур ОАО "ИТКОР", М:, 2012.

4. Струбалин П.В. Учет и анализ движения и использования программных продуктов Автореф. дис. канд. экон. наук. - Саратов, 2003. - 18 с.

5. Струбалин П.В. Учет и анализ движения и использования программных продуктов дис. канд. Экон., наук. - М.: 2002. - . - Саратов, 2003. 80-85 с.

6. Струбалин П.В., Фролова Н.Б. Информационные технологии, обеспечивающие работу и безопасность систем электронного дистанционного обучения Информационная безопасность регионов. 2015. № 4 (21). C. $12-18$.

\title{
Егоров. А.Н., Кузнецов В.А. \\ Параллельный подход к решению задачи оптимизации оперативного управления транспортным конвейером шлюзованной системы
}

Государственный университет морского и речного флота имени адмирала С.О. Макарова (Россия, Санкт-Петербург)

doi:10.18411/lj-31-03-2018-13

idsp: 000001:lj-31-03-2018-13

\section{Аннотация}

В статье рассматривается задача оптимизации оперативного управления транспортным конвейером шлюзованного судоходного канала. Предлагается параллельный алгоритм её решения.

Ключевые слова: оптимизация оперативного управления, метод ветвей и границ, параллельные алгоритмы.

В статье [1] рассматриваются вопросы оптимизации оперативного управления транспортным конвейером шлюзованного судоходного канала (ШСК). Обосновывается возможность реализации автоматизированной диспетчеризации судопропуска на основе взаимодействия имитационной модели и алгоритма управления в реальном масштабе времени, конечной целью которой является расписание проводки судов. До настоящего времени подобных систем, работающих в рамках шлюзованного судоходного канала в целом, не создано. Делается вывод, что для этого необходимо использовать системы, обеспечивающие автоматический обмен информацией между судами и береговыми службами, а также параллельные алгоритмы обработки данных.

Дадим обобщённое описание задачи оптимизации оперативного управления транспортным ШСК, формализация которой представлена в [2].

Пусть задан период планирования [t0,t0+T], где $\mathrm{t} 0$ - начало периода планирования, a T - горизонт планирования. Пусть $\mathrm{N}$ - количество объектов ШСК, выполняющих обслуживание судов при прохождении канала, а $\mathrm{M}$ - количество судов находящихся на ШСК в период планирования. Тогда расписанием R функционирования ШСК на период планирования $[\mathrm{t} 0, \mathrm{t} 0+\mathrm{T}]$ назовём таблицу размерности $\mathrm{M}^{*} \mathrm{~N}$, в которой каждый элемент $(\mathrm{i}, \mathrm{j})-$ совокупность $\left(\tau_{\mathrm{ij}}^{\mathrm{H}}, \tau_{\mathrm{ij}}^{\mathrm{K}}\right)$, где, $\tau_{\mathrm{ij}}^{\mathrm{H}}-$ время начала, a $\tau_{\mathrm{ij}}^{\mathrm{K}}-$ время окончания выполнения технологической операции на объекте ј над i-й группой судов.

Требуется на период планирования [t0,t0+T] сформировать такое расписание $\mathrm{R}$, которое обеспечивает наилучшее значение выбранного критерия оптимизации и удовлетворят множеству естественных и технологических ограничений.

Для решения задачи в [2] предлагается алгоритм основанный на методе ветвей и границ, принцип работы которого заключается в имитации работы системы в период планирования [t0,t0+T], построении дерева всех возможных вариантов управления на 
заданном интервале и выборе наилучшего управляющего воздействия по заданным критериям. Для этого используется имитационно-управляющая система (ИУС) ШСК, которая содержит в своем составе имитационную модель функционирования ШСК, осуществляющую генерацию допустимых вариантов управления и имитационное моделирование поведения системы при принятии этих вариантов.

Для сокращения числа перебираемых вариантов используется метод «отсечения» неперспективных решений, приводящих к недопустимой ситуации в системе, или не обеспечивающих заданный уровень устойчивости. Следует отметить, что «отсечение» недопустимых ситуаций выполняет имитационная модель.

Формирование дерева вариантов управления является многошаговым рекурсивным процессом. Каждый узел дерева рассматривается как некоторое состояние системы S в момент времени t. Генерация дерева начинается с его корня, который представляет собой начальное состояние системы в момент $\mathrm{t}=\mathrm{t} 0$. При продвижении по дереву для каждого узла выполняются следующие действия:

1. Имитация работы системы из исходного состояния $\mathrm{S}$ до состояния $\mathrm{S}$ ' момента времени $t^{\prime}>t$ при котором необходимо принять управляющее решение.

2. Оценка устойчивости расписания для состояния $\mathrm{S}^{\prime}$, если значение не удовлетворяет требуемому уровню то данная ветвь в дальнейшем не рассматривается и происходит возврат к последней точки ветвления.

3. Для состояния S' строиться множество возможных вариантов управления $\{\mathrm{Ui}\}=\{\mathrm{U} 1, \mathrm{U} 2, \ldots, \mathrm{UN}\}$, если вариантов больше 1 то на дереве образуется точка ветвления.

4. Далее рассматривается только один вариант управления, который применяется в текущему состоянию S', после чего алгоритм выполняется заново для состояния $\mathrm{S}^{\prime}(\mathrm{Ui})$ в момент времени t'. Остальные варианты управления запоминаются до возвращения алгоритма к этой точке ветвления.

5. При достижении конца периода планирования $\mathrm{t}^{\prime}>=\mathrm{t} 0+\mathrm{T}$, выполняется оценка критерия оптимизации сформированного управляющего воздействия, которое представляет собой совокупность решений принятых при продвижении от начального состояния системы в момент t0 до текущего состояние $S$ ' момента t'. Если оно лучше текущего оптимального управления, то становиться им. При этом сохраняется и достигнутое состояние S'. После чего алгоритм возвращается к предыдущей точки ветвления, где рассматривается следующее по очереди управляющее воздействия данной точки. При отсутствии нерассмотренных альтернатив в данной точки алгоритм переходит к более ранней точке ветвления и т.д. до достижения корневого узла.

После проверки всех возможных вариантов управления из сохранённого состояния $\mathrm{S}$ ' формируется расписание $\mathrm{R}$, которое является оптимальным на заданном периоде планирования.

Основной задачей при переходе от последовательного алгоритма к параллельному является разбиение исходной задачи на независимые подзадач.

Формально дерево вариантов управления представляет собой конечное множество $\mathrm{L}$, состоящей из одного или более узлов, таких что имеется один специально обозначенный узел, называемый корнем дерева, а остальные узлы, исключая корень, содержатся в $\mathrm{m}>=1$ попарно непересекающихся множеств L1, L2, ... Lm, каждое из которых, является деревом. Следовательно, в качестве подзадачи можно рассматривать поиск оптимального управления в одном из попарно не пересекающихся множеств поддеревьев.

Согласно закону Амдаля - врем выполнения параллельного алгоритма не может быть меньше выполнения самого длинного его фрагмента, т.е. для достижения максимальной эффективности при распараллеливании необходимо сформировать подзадачи так, чтобы для каждого потока время вычисления было примерно одинаково. 
Время проверки дерева вариантов определяется количеством узлов в нём. Пусть $\mathrm{G}$ это среднее количество ветвлений для каждого узла дерева, тогда максимальное количество узлов в дереве определяется как $\sum \mathrm{Gd}$, где $\mathrm{d}=0 \ldots \mathrm{D}$ - уровень глубины дерева, a D - количество уровней глубина дерева. Следовательно, для того чтобы два поддерева из одного дерева имели равное количество узлов, необходимо, чтобы их корни располагались на одном уровне глубины.

Согласно описанному алгоритму работы ИУС ШСК после обработки одного узла дерева вариантов управлений на следующем этапе рассматривается узел располагающийся на следующим уровне глубины дерева. В результате такого продвижения, конечные узлы дерева располагаются на различных уровнях глубины. Назовём такой характер перемещения по дереву вертикальным (рис. 1,a). При горизонтальном движении (рис. 1,б) выполняется последовательное прохождение каждого узла текущего уровня глубины, после чего осуществляется переход на следующий уровень, при этом в любой момент времени все конечные узлы дерева располагаются на одном или соседних уровнях глубины.

Алгоритм работы ИУС ШСК в режиме горизонтального прохождения узлов отличается от вертикального лишь тем, что после формирования множества возможных вариантов управления \{Ui\} для состояния S', варианты не рассматриваются сразу, а сохраняются на следующем уровне дерева управления для дальнейшей обработки. Затем алгоритм переходит к следующему узлу, находящемуся на том же уровне глубины или первому узлу следующего уровня. При этом движение к предыдущей точки ветвления не осуществляется, т.к. все не рассмотренные варианты управления могут находиться только на следующих узлах. Учитывая, что все основные этапы работы алгоритма сохранены то скорость работы ИУС ШСК при вертикальном и горизонтальном характере движения в общем случае можно считать равной.
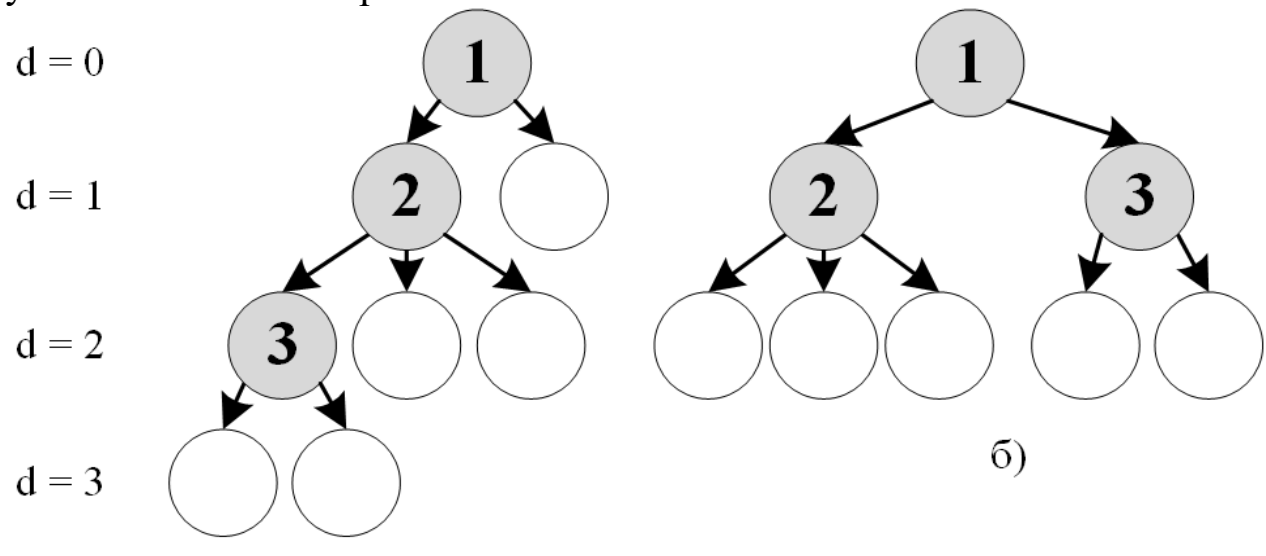

б)

a)

Рис. 1. Структура дерева после обработки трёх узлов при различном характере движении по дереву: а) вертикальныці; б) - горизонтальнылй

Проверка управляющего воздействия осуществляется при достижении конца планирования на некотором уровне d. При увеличении уровня глубины дерева количество его узлов увеличивается для вертикального характера движения линейно $\mathrm{G} * \mathrm{~d}$, а для горизонтального экспоненциально Gd. Из чего следует, что алгоритм работы ИУС ШСК в режиме вертикального прохождения узлов, при прочих равных условиях, расходует значительно меньше памяти, по сравнению с горизонтальным.

После сравнения эффективности алгоритмов можно сделать вывод, что для формирования подзадач, при параллельной обработке, необходимо использовать алгоритм работы ИУС ШСК в режиме горизонтального прохождения узлов, а для поиска оптимума в каждой из подзадач на отдельном потоке более предпочтительным является вертикальный режим работы ИУС. 
Пусть многопоточная система имеет К параллельно работающих потоков, тогда алгоритм параллельной работы ИУС ШСК можно описать следующим образом.

Этап 1. Выполнение алгоритма работа ИУС в режиме горизонтального прохождения узлов до тех пор, пока количество конечных узлов древа вариантов управления не будет максимально близким к числу К, но не превысит его.

Этап 2. Каждый конечный узел, сформированного дерева, отправляется на отдельный поток для дальнейшей обработки.

Этап 3. На каждом потоке выполняется поиск оптимума для поддерева вариантов управления переданной подзадачи. Для этого используется алгоритм работы ИУС в режиме вертикального прохождения узлов.

Этап 4. После завершения расчётов каждый поток возвращает оптимум соответствующей подзадачи из совокупности, которых выбирается наиболее оптимальный вариант.

При распараллеливании последовательного алгоритма работы ИУС ШСК размерность задачи, обрабатываемой на одном из множества параллельно работающих потоков, была снижена не менее чем в К раз, что, если не учитывать временные затраты на синхронизацию потоков, обеспечивает прирост производительности в порядка К раз по сравнению с последовательной реализацией.

$$
* * *
$$

1. Егоров А.Н., Кузнецов В.А. Распараллеливание вычислений для автоматизированной диспетчеризации судопропуска шлюзованной системы // Вестник Государственного университета морского и речного флота имени адмирала С. О. Макарова. — 2016. — № 2 (36). - С. 214-224.

2. Бутов А.С., Гаскаров Д.В., Егоров А.Н., Крупенина А.Н. / под общ. ред. проф. Бутова А.С. - СПб.: Судостроение, 2001. - $552 \mathrm{c}$.

\section{Иванов Е. Д., Гриценко Е.М. \\ Разработка мобильного приложения для проведения опросов в сервисных центрах}

(Россия, Красноярск)

doi:10.18411/lj-31-03-2018-14

idsp: 000001:lj-31-03-2018-14

\section{Аннотация}

В статье описаны этапы разработки мобильного приложения на платформе Android и технические характеристики системы.

Ключевые слова: мобильное приложение, программное обеспечение, система, сервер, платформа, качество услуг.

За прошедшие два десятилетия мобильные технологии изменили мир и оказали влияние на информационное общество, улучшив производство, хранение и обработку информации. Обладатель мобильного устройства способен воспользоваться услугами банка, заказом такси или доставки еды, электронной почтой, личным кабинетам и др. С каждым годом появляются новые модели, улучшаются стандарты обмена данными и версии мобильных операционных систем. Лидеры мобильного рынка, к которым относятся Samsung и Apple, производят смартфоны, планшеты и smart-часы, оснащенные производительными аппаратными характеристиками и современными операционными системами, OC Android и $\mathrm{OOS}$ соответственно. На рисунке 1 приведена статистика популярности.

Преимущество мобильных приложений - в их использовании на личном устройстве пользователей. Пользуясь мобильными приложениями, потребители заинтересованы в использовании услуг компаний, совершении покупок, а также в 